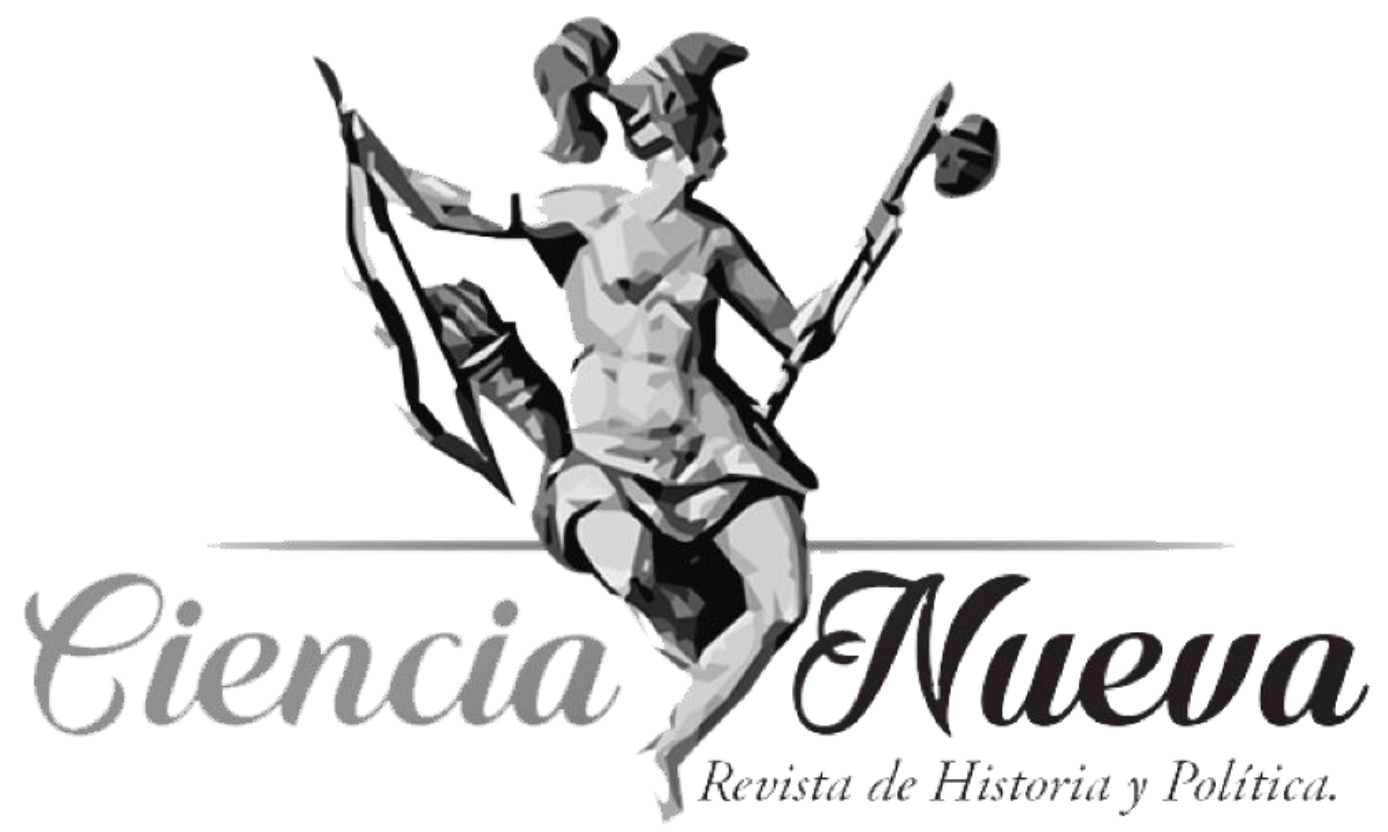

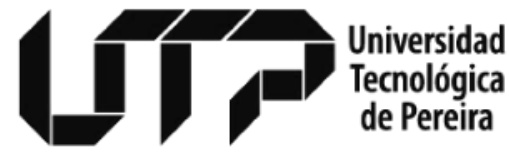

Maestría en Historia

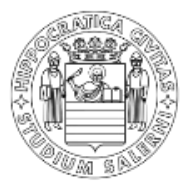

UNIVERSITÀ DEGLI STUDI DI SALERNO

Maestría en Ciencia Política

CIENCIAS POLÍTICAS

\title{
LA ROVENTE ESTATE DEL ‘43 QUANDO MUSSOLINI, HITLER, ROOSEVELT E CHURCHILL DECISERO IL DESTINO \\ DELL'ITALIA
}

EL VERANO TURBULENTO DE 1943 CUANDO MUSSOLINI, HITLER, ROOSEVELT Y CHURCHILL DECIDIERON EL DESTINO DE ITALIA

Ugo della Monica pp. 119-132

Vol. 2 Núm. 1, Enero-Junio de 2018

Pereira, Colombia 


\section{LA ROVENTE ESTATE DEL '43 QUANDO MUSSOLINI, HITLER, ROOSEVELT E CHURCHILL DECISERO IL DESTINO DELL'ITALIA* \\ EL VERANO TURBULENTO DE 1943 CUANDO MUSSOLINI, HITLER, ROOSEVELT Y CHURCHILL DECIDIERON EL DESTINO DE ITALIA}

Ugo della Monica**

udellamonica@unisa.it

ORCID: https://orcid.org/0000-0001-9120-6503

\begin{tabular}{rlr}
\hline Recibido: & 23 de octubre de 2017 \\
Revisado: & 28 de noviembre de 2017 \\
Aceptado: & 25 de enero de 2018 \\
Publicado: & 12 de marzo de 2018 \\
\hline
\end{tabular}

\section{Riepilogo:}

L'articolo propone una lettura, attraverso l'analisi di documenti nuovi ed inediti, del periodo storico che intercorre dall'inizio di luglio del 1943 alla fine di settembre dello stesso anno: periodo in cui l'Italia vede il cambio di rotta della guerra che si trasforma da guerra alleata alla Germania, contro le forze angloamericane, a guerra combattuta contro la stessa Germania e che vede nell'Italia una disastrosa sconfitta. Attraverso l'analisi degli atti diplomatici inglesi, americani, russi, tedeschi ed italiani si evince come realmente si è conclusa la parentisi del Ventennio e, soprattutto come si è giunti alla firma dell'armistizio.

\section{Parole chiave:}

Guerra; Armistizio, Mussolini, Churchill, Roosevelt.

\section{Resumen:}

El artículo propone una lectura, a través del análisis de documentos nuevos e inéditos, del período histórico entre principios de julio de 1943 hasta finales de septiembre del mismo año. Período en el que Italia ve el cambio de curso de la guerra que se transforma de una guerra aliada con Alemania contra las fuerzas angloamericanas, a la guerra contra la propia Alemania y que ve en Italia una derrota desastrosa. A través del análisis de las acciones diplomáticas británicas, americanas, rusas, alemanas e italianas, queda claro el final del siglo Xx y, sobre todo, cómo se firmó el armisticio.

\section{Palabras clave:}

Guerra; Armisticio, Mussolini, Churchill, Roosevelt.

\footnotetext{
* El presente artículo respeta las directrices y normas dispuestas en la Declaración de Ética de Publicación de Ciencia Nueva, Revista de Historia y Política. Esta declaración puede consultarse en la página web de la revista: revistas.utp.edu.co/index.php/historia

** Dottorato in Teoria e Storia delle Istituzioni e Dottorato di Ricerca dei Dipartimenti di Studi Umanistici dell'Università Degli Studi di Salerno, Italia.
} 
$\mathrm{I}$ n queste pagine ho voluto ripercorrere, in una sorta di memoria, quei mesi cruciali per il destino del nostro Paese che vanno dall'inizio di luglio alla fine di settembre del 1943. Mesi in cui la guerra a fianco dell'alleato germanico si trasformò nella guerra combattuta contro il nemico germanico e che vide per l'Italia una disastrosa disfatta. Il popolo italiano si trovò travolto, grazie alle decisioni e soprattutto alle scelte altrui, in un vortice che nel giro di una stagione lo avrebbe completamente inghiottito, senza avere nemmeno il tempo di capire cosa realmente stesse accadendo. Sono i mesi in cui si è passati dall'essere fascisti sicuri al diventare antifascisti convinti; mesi in cui ci si è visti uccidere dai compagni tedeschi, alleati da anni; mesi in cui i nemici angloamericani, si sono trasformati in quei generosi amici, prodighi di quelle leccornie che mai sino ad allora erano state gustate. Non è stato certamente facile per gl'italiani di allora attraversare e superare questo breve ma intenso periodo che è risultato essere cruciale per il prosieguo di quella guerra che si pensava fosse terminata prima il 25 luglio e poi l'8 settembre e che invece durò ancora a lungo.

Oggi è sempre più raro, ma ancora possibile, incontrare quei ragazzini di allora che, nonostante siano trascorsi oltre settant'anni, con lucida memoria ricordano ogni singolo momento, ogni singolo evento e finanche ogni singolo messaggio radiofonico con cui venivano annunciati gli eventi che si stavano vivendo. Per tutti coloro che, come me, non hanno vissuto quel periodo, propongo la lettura di questo "diario", rimanendo fedele ai fatti e soprattutto evitando giudizi e commenti personali. Scrivere questa memoria di guerra è stato possibile grazie al fatto che, trascorsi settant'anni, imposti dalla legge sulla privacy per i documenti d'archivio, go potuto consultare e studiare presso l'Archivio Centrale dello Stato a Roma, le carte dell'epoca, corredandole con quelle degli eserciti alleati che in specifici casi si consultano direttamente on-line.

\section{Il tradimento del Patto ed il crollo del Fascismo}

La guerra lampo ${ }^{1}$ che Mussolini si era affrettato a dichiarare al Mondo dal balcone di Palazzo Venezia, il 10 giugno del $1940^{2}$, dopo aver arrecato distruzione, morte e depressione nel popolo e nel Paese tutto, sembrò prendere una differente piega nell'estate del 1943 quando tutti i grandi centri erano stati duramente colpiti riportando ingenti danni; gli italiani, dal canto loro, sia fascisti che antifascisti, lealmente avevano combattuto per tre lunghissimi anni, al fianco degli alleati tedeschi in difesa del Paese pur sapendo, da principio, che alla fine tutto sarebbe risultato vano; difatti, molti erano coscienti, da subito, che la loro era una causa sbagliata e che le prospettive della rapida vittoria credute dal Duce, erano ormai svanite. La guerra divenne sempre meno popolare, come lo stesso Mussolini al quale si iniziavano ad addossare tutti gli errori bellici, tattici e di valutazione generale che avevano portato l'Italia all'ultimo posto tra le potenze in conflitto.

In effetti, la complessa situazione politico-militare nazionale mutò radicalmente rotta a partire dal 10 luglio del 1943 quando le forze angloamericane avviano lo sbarco sulle coste siciliane; il 13 le truppe italiane, di stanza in diversi luoghi dell'isola, ripiegano: la divisione

\footnotetext{
1 «Mussolini, sicuramente, senza la sconfitta britannica non sarebbe entrato in guerra, vi entrò in solamente perché spinto dalla paura della Germania». Sono queste le parole del triumviro Dino Grandi. Archivio Centrale dello Stato - Archivio Grandi, b. 152, fasc. 199, sottofascicolo 6, 1 agosto 1944, f.86.

${ }^{2}$ All'atto della consegna della dichiarazione di guerra, da parte del Ministro degli Esteri Ciano, l'ambasciatore francese Poncet commentò «E così, avete aspettato di vederci in ginocchio, per accoltellarci alle spalle». Il dibattito sulle parole dell'ambasciatore francese è tutt'oggi di attualità tanto che, nel 2009 fu il tema principale di un convegno organizzato a Roma, nella sede di Palazzo Farnese, dallo storico-politologo Maurizio Serra.
} 
"Napoli", dopo una strenua resistenza, viene completamente distrutta; Augusta, Gela, Licata ed il litorale di Siracusa cadono in mano alle forze alleate. In effetti l'azione militare alleata, per certi punti di vista, è paragonabile ad un naturale passaggio di comandi, simile a quello avvenuto decenni prima con l'arrivo di Garibaldi: chissà se gli astuti e scaltri americani non avessero studiato, oltre alla posizione strategico-geografica della centralità europea dell'isola, la storia e l'indole del popolo siculo, da sempre pronto a cambiar casacca e padrone.

Nella notte tra il 13 e il 14 luglio il nemico, esaltato dai successi ottenuti, opera un'ulteriore sbarco grazie a centinaia di paracadutisti minacciando lo stretto di Messina che, successivamente, capitola il giorno 17. Il 19, invece, sul versante continentale, per la prima volta da quando è scoppiato il conflitto, viene bombardata la "Città Santa" ritenuta da tutti inattaccabile per la presenza del Papa: furono colpiti i centri ferroviari ed i quartieri Tiburtino, San Lorenzo e Porta Maggiore, la città Universitaria, gli aeroporti del Littorio e di Ciampino; fu distrutta, quasi del tutto, la Basilica di San Lorenzo. Per la prima volta da quando, nel 1939 era salito al soglio di Pietro, Pio XII lascia le mura vaticane per recarsi sui luoghi del massacro, facendo ben attenzione a non incrociarsi con il re, accorso anch'esso sui luoghi disastrati, onde evitare inconvenienti diplomatici.

Il 19 luglio il Duce, che ormai non sa più cosa aspettarsi dalla Germania e che soprattutto è ben cosciente che la situazione gli è completamente scivolata di mano, incontra, con vane ed inutili aspettative, l'amico Hitler a Feltre ${ }^{3}$, avanzandogli una serie di richieste, senza però riuscirne ad ottenerne nessuna: né un aeroplano, né un carro armato, né una divisione; in effetti la cattiveria e l'astuzia del Fuhrer, che aveva ben capito la situazione tattico-politica italiana, costringe Mussolini a firmare il patto Alarico, che prevedeva il passaggio in Italia di forze tedesche e che in seguito vedremo in azione.

La mattina del giorno 21 il triumviro Dino Grandi - che assieme a Galeazzo Ciano, Luigi Federzoni, Carlo Sforza, Alfredo De Marsico e Giuseppe Bottai stanno studiando e tramando su come e quando sbarazzarsi di Mussolini, redigendo quello che sarebbe passato alla storia come l'ultimo Ordine del giorno dell'ultima riunione del Gran Consiglio del Fascismo - telegrafa all'aiutante di Campo del Re, Generale Paolo Puntoni, chiedendogli di far aprire gli occhi al re comunicandogli quanto stava accadendo e ricordandogli, soprattutto, che:

A quasi cent'anni dal giorno in cui Re Carlo Alberto emanò lo Statuto del Regno e iniziò, col Risorgimento, la lotta per la libertà, l'unità e l'indipendenza d'Italia, la Patria va oggi verso la disfatta e il disonore ${ }^{4}$.

Vittorio Emanuele, ascoltato Puntoni in proposito convoca per il giorno successivo, in seduta straordinaria, il Duce al fine che si riesca insieme a prospettare nuovi programmi ma soprattutto una nuova strategia tattico-militare e di governo capace di far uscire il Paese da quella situazione di impasse, in cui brancolava da mesi. L'incontro si concluderà con un nulla di fatto in quanto, alla fine dell'udienza, congedato Mussolini, il re commenta con lo stesso Puntoni:

\footnotetext{
${ }^{3}$ Documenti del Ministero della Difesa dell'ex URSS, divenuti pubblici nel 2009, comproverebbero che in questa circostanza era previsto, ed anche già organizzato, il duplice omicidio di Mussolini ed Hitler annullato, all'ultimo momento, da un contrordine del Vaticano a cui, nonostante tutto, continuava a far paura l' «avanzata rossa».

${ }^{4}$ Cfr. Paolo Puntoni, Parla Vittorio Emanuele III (Bologna: Il Mulino, 1993), 142.
} 
Ho tentato di far capire al Duce che ormai soltanto la sua persona, bersagliata dalla propaganda nemica e presa di mira dalla pubblica opinione, ostacola la ripresa interna e si frappone a una definizione netta della nostra situazione militare. Non ha capito o non ha voluto capire. È come se avessi parlato al vento ${ }^{5}$.

Momento fondamentale e decisivo per la successiva sorte del Paese, tanto nell'immediato, quanto per gli anni a venire, è determinato da tutto ciò che accadde nella notte del 24 luglio quando il Gran Consiglio, votando l'ordine Grandi, esautora Mussolini.

La congiura che portò al voto di sfiducia Mussolini, appare oggi, un errore politico rilevante che squalificò per sempre quei responsabili che avevano votato contro il loro Duce. Che cosa pensavano infatti? Che il re li avrebbe incaricati di sostituire Mussolini? In effetti si sono fatti "giocare" da Vittorio Emanuele e dal vecchio ufficiale Badoglio che non aveva mai brillato per soverchia astuzia, così come vedremo negli avvenimenti successivi. $O$ forse pensavano solo di uscirne nel modo più vantaggioso? Quest'ultima ipotesi è ancora più disonorevole!

Dopo la conclusione della seduta, alle 3,30, Grandi incontra il Ministro della Real Casa Acquarone per informarlo dell'esito della votazione e lo incarica di comunicare al Re che tutto ora dipendeva dalla rapidità e dal coraggio con cui avrebbe egli agito. In effetti la rapidità di Vittorio Emanuele, nonostante la sua abulica pacatezza, è talmente vivida che, non attendendo nemmeno l'esito della votazione del Gran Consiglio, aveva già conferito al generale Badoglio l'incarico di formare il nuovo governo, in una Roma in totale stato d'assedio.

La sorte di Mussolini, in effetti, il re la stava architettando già da diversi giorni, la qual cosa si evince dalla risposta che il Generale dell'Esercito Ambrosio fornisce al Generale dei Carabinieri Cerica nel momento in cui questi gli chiede la costituzionalità dell'operazione di villa Savoia, egli risponde: "l'ordine viene dal Sovrano".

Il generale Puntoni, giunto alle 10,50 del 25 a villa Savoia, trova il re "[...] tranquillo e sereno e dalle parole di Sua Maestà mi è facile capire che ormai la situazione di Mussolini è stata decisa. Il sovrano affronterà il Duce domani, lunedì, durante la consueta relazione"6. È, invece, lo stesso Mussolini che richiede di anticipare l'udienza per le ore 17,00 di quello stesso giorno, richiesta che viene immediatamente accettata, tanto che il Duce viene prontamente ricevuto, in forma privata, a Villa Savoia, da Vittorio Emanuele III che, per la prima volta dopo ventuno anni, riprende in mano il potere "regio" e fermamente comunica a Mussolini, senza farsi interrompere nel discorso e senza acconsentire alcuna lagnanza, di averlo sostituito, nella carica di presidente del Consiglio, con il maresciallo Badoglio e per di più, al fine di salvaguardare la sua incolumità, si vede costretto a metterlo "sotto protezione". In effetti, il re agisce come se nulla di così eclatante stesse accadendo, per lui, risulta essere un normale passaggio di consegne. Mussolini, invece, ricorderà di questo momento, successivamente:

... il colloquio che io ebbi col re a Villa Savoia durò venti minuti e forse meno. Trovai un uomo col quale ogni ragionamento era impossibile poiché egli aveva già preso le sue decisioni; lo scoppio della crisi era imminente. È già accaduto, in pace e in guerra, che un ministro sia dimissionario, un comandante silurato, ma è un fatto unico nella storia che un

\footnotetext{
${ }^{5}$ Cfr. Paolo Puntoni, Parla Vittorio Emanuele III...

${ }^{6}$ Cfr. Paolo Puntoni, Parla Vittorio Emanuele III ..., 143.
} 
uomo il quale, come colui che vi parla, aveva per ventuno anni servito il re con assoluta, dico assoluta lealtà, sia fatto arrestare sulla soglia della casa privata del re, costretto a salire su una autoambulanza della Croce Rossa col pretesto di sottrarlo ad un complotto e condotto a tutta velocità prima in una e poi in un'altra caserma dei carabinieri. Ebbi subito l'impressione che la protezione non era che in verità che un fermo ${ }^{7}$.

La calma con cui il Duce, ormai stanco ed avvilito dal tradimento dei suoi "compagni", ascolta e accetta la decisione del re è inversamente proporzionale all'ira scatenatasi da parte della regina Elena che non ha minimamente accettato il fatto che il marito abbia fatto arrestare Mussolini nella loro residenza privata, per di più, con un'imboscata architettata nei minimi particolari; fermamente sosterrà che l'arresto doveva avvenire nei palazzi di potere e non in casa ed alla presenza del Sovrano.

Dall'altro versante politico, quello anglo-americano, venuto a conoscenza della caduta di Mussolini, Roosevelt scrive al premier britannico Churchill: "La popolazione italiana deve essere trattata bene, ma il capo dei diavoli (Mussolini) deve esserci consegnato insieme con i principali complici dei suoi crimini".

Churchill in risposta:

Se i capi fascisti cadessero nelle nostre mani decideremo come trattarli consultandoci con gli Stati Uniti e, dopo esserci accordati con loro, con l'Unione Sovietica. Qualcuno potrebbe preferire una esecuzione immediata e senza processo, salvo che per scopi di identificazione. Altri preferirebbero che fossero segregati fino alla fine del conflitto in Europa e che la loro sorte fosse decisa insieme con quella degli altri criminali di guerra. Personalmente sono abbastanza indifferente su questo punto, purché nessun solido vantaggio militare sia sacrificato a una vendetta immediata ${ }^{8}$.

Alle 22,45 dalla radio giungono all'orecchio incredulo e disorientato del popolo italiano le parole del re: "Nell'ora solenne che incombe sui destini della patria ognuno riprenda il suo posto di dovere, di fede e di combattimento: nessuna deviazione deve essere tollerata, nessuna recriminazione essere consentita".

Il maresciallo Badoglio annunciando l'assunzione dei pieni poteri afferma: "La guerra continua, l'Italia duramente colpita nelle sue province invase, nelle sue città distrutte, mantiene fede alla parola data, gelosa custode delle sue millenarie tradizioni"9.

Il giorno seguente, con un più che falso atto di stima, Badoglio comunica a Mussolini:

Il sottoscritto, capo del governo, tiene a far sapere a Vostra Eccellenza che quanto è stato eseguito nei Vostri riguardi, è unicamente dovuto al Vostro personale interesse, essendo giunte da più parti precise segnalazioni di un serio complotto contro la Vostra persona. Spiacente di questo, tiene a farVi sapere che è pronto a dare ordini per il Vostro sicuro accompagnamento, con i dovuti riguardi, nella località che vorrete indicare ${ }^{10}$.

\footnotetext{
${ }^{7}$ Cfr. Benito Mussolini, “Discorso di Monaco del 18 settembre 1943”, Meridiano d'Italia illustrato, 12 marzo 1952.

${ }^{8}$ Cfr. Roosevelt Churchill, Carteggio segreto di guerra, Francis Loevenheim, Harold D. Langley e Manfred Jones (a cura di) (Milano: Mondadori, 1977), 45.

${ }^{9}$ Proclami di Vittorio Emanuele III e di Pietro Badoglio, pubblicati in un volumetto, in mio possesso, La verità, senza data e senza editore, contenente differenti proclami.

${ }^{10}$ Cfr. Benito Mussolini, Scritti e discorsi di Benito Mussolini (Milano: Hoepli, 1934).
} 
Mussolini, dal canto suo, con altrettanta finta stima, risponde a Badoglio: "Desidero assicurare il Maresciallo Badoglio, anche in ricordo del lavoro in comune svolto in altri tempi, che da parte mia non solo non gli verranno create difficoltà di sorta, ma sarà data ogni possibile collaborazione"11.

L'italico popolo fascista, nell'arco di ventiquattrore, spazzò via gli ultimi vent'anni di vita pubblica e privata del Paese, rinnegando sin da subito di aver creduto, adulato ed osannato l'uomo, il politico, lo statista, il condottiero e lo stratega Mussolini. La giornata del 26 luglio, infatti, risultò essere una delle più lunghe e dure di tutto il periodo in quanto si dovettero bloccare quelle rivolte, tanto fasciste quanto antifasciste, che provocarono centinaia di morti in tutto il Paese. Si registrarono eventi sconcertanti a Roma a Savona a Milano ed in altre città dove riuscirono ad evadere dal carcere centinaia di detenuti creando paura, disordine e insubordinazione ad un apparato statale ormai del tutto inesistente ma soprattutto al totale sbando. In questo clima di smobilitazione della vita sociale, politica, civile ed istituzionale del Paese, il Comando supremo germanico, avendo subdorato che l'Italia stesse tramando e trattando con gli Alleati da inizio alla succitata operazione Alarico che, stabilita nell'incontro di Feltre, prevede il trasferimento in territorio italiano di nuove truppe tedesche attraverso il valico del Brennero, senza il bisogno di alcuna autorizzazione dai comandi italiani e, soprattutto, senza doverne specificare la destinazione: da questo momento i tedeschi risultano esser veri e propri cani sciolti; con la suddetta operazione, poterono contare, da subito sul territorio italiano, di un esercito composto da 17 divisioni, di cui 7 motorizzate, diverse corazzate, oltre a 150000 soldati non inquadrati nelle divisioni, la qual cosa si trasformerà nella definitiva catastrofe per l'Italia.

\section{Pedine in mano degli Alleati}

Dall'altro lato dell'Oceano, quella che sembrava essere la trattativa di armistizio tra Italia e forze alleate iniziava ad avere una sua veridicità e validità. Nel corso di una telefonata, Churchill comunica a Roosevelt: "Non vogliamo che si facciano proposte di armistizio prima che siano presi contatti con noi. Possiamo anche aspettare senza muoverci un giorno o due". Il presidente americano si dichiara d'accordo. La telefonata viene intercettata dai tedeschi che commentano: "Ecco la prova irrefutabile che sono in corso trattative segrete fra gli angloamericani e l'Italia".

Il 29 luglio è trasmesso alla radio un messaggio del generale Eisenhower agli italiani:

Noi ci compiacciamo col popolo italiano e con casa Savoia per essersi liberati di Mussolini, l'uomo che li ha coinvolti in guerra come strumento di Hitler e li ha portati sull'orlo del disastro. Il più grande ostacolo che divideva il popolo italiano dalle Nazioni unite è stato rimosso dagli italiani stessi. [...]. Noi veniamo come liberatori. Il vostro ruolo consiste nel cessare immediatamente ogni assistenza alle forze armate tedesche nel vostro paese. Se farete ciò, noi vi libereremo dai tedeschi e dagli orrori della guerra. Come avete già visto in Sicilia, la nostra occupazione sarà mite e benefica.[...] Le antiche libertà e tradizioni del vostro paese saranno ristabilite ${ }^{12}$.

\footnotetext{
${ }^{11}$ Cfr. Benito Mussolini, Scritti e discorsi di Benito Mussolini...

12 Cfr. Mario Avagliano, Il partigiano Montezemolo: storia del capo della resistenza militare nell'Italia occupata (Milano: Dalai editore, 2012), 158.
} 
Sempre Eisenhower, a soli due giorni dall'elogio agli italiani, visto che Badoglio ancora non riusciva a prendere alcuna decisione in merito all'armistizio, invia un ulteriore messaggio radio agli italiani dal tono totalmente differente rispetto al precedente e tutt'altro che pacato:

Italiani, vi mandiamo un monito solenne, il periodo di pausa è finito. Siate pronti. Presto inizierà l'offensiva aerea senza remissione di giorno e di notte e quando cadranno le bombe ricordatevi che il sangue sparso di ogni italiano cade sulle mani degli uomini di Roma che, nell'ora in cui l'Italia dovrebbe decidere, hanno temporeggiato.

Churchill, dal canto suo e soprattutto da sempre più accomodante, scrive al presidente americano Roosevelt: "Tratterò con qualunque autorità italiana che possa darci quello che vogliamo. Non ho la minima paura di dare l'impressione di riconoscere Casa Savoia e Badoglio, purché facciano fare agli italiani quello che serve ai nostri obiettivi di guerra"13.

L'Italia in questo momento è solamente una pedina in mano ad esperti e risoluti giocatori che sanno per certo che vinceranno la guerra e che, senza scrupolo alcuno, inviano un ulteriore messaggio radio agli italiani:

Otto giorni sono passati e Badoglio temporeggia ancora. Vi avvisiamo ancora: la nostra aviazione vi colpirà dal cielo e ben presto le nostre forze terrestri combatteranno sul vostro territorio. Abbiamo atteso; vi abbiamo avvisato. Non abbiamo scelta. Non possiamo far altro $^{14}$.

Tra il 12 ed il 13 agosto, infatti, l'aviazione alleata bombarda Milano e Torino. A Milano, 405 bombardieri Lancaster e Stirling sganciano sulla città 1.904 tonnellate di bombe; a Roma, l'aviazione angloamericana colpisce i quartieri Tiburtino, Prenestino, Casilino e Tuscolano; vengono colpite diverse altre città. Il maresciallo Badoglio, il giorno 13 dichiara unilateralmente Roma "città aperta" priva, cioè, di comandi militari e di qualsiasi altro obiettivo che possa giustificare i bombardamenti alleati. Gli alleati disconoscono la decisione. Le direttive di Churchill sulla resa senza condizioni sono:

Il maresciallo Badoglio deve capire che non possiamo trattare, ma solo esigere la resa incondizionata, il che significa che il governo italiano dovrebbe porsi nelle mani dei governi alleati i quali poi detteranno le loro condizioni saranno tali da imporre agli italiani una capitolazione onorevole ${ }^{15}$.

Il ministro degli Esteri, Raffaele Guariglia, invece, intende fermamente trattare la resa solo a condizione che l'Italia sia considerata paese alleato la qualcosa, non piacendo affatto agli alleati, crea una interruzione nella trattativa. Il 16 agosto le truppe angloamericane completano l'invasione della Sicilia, occupando Messina. Il maresciallo britannico Alexander comunica al primo ministro Churchill:

\footnotetext{
${ }^{13}$ Cfr. Roosevelt Churchill, Carteggio segreto di guerra...,62.

${ }^{14}$ Cfr. Mario Avagliano, Il partigiano Montezemolo..., 162.

${ }^{15}$ Cfr. Elena Aga Rossi, L'inganno reciproco. L'armistizio tra l'Italia e gli angloamericani del settembre 1943 (Roma: Pubblicazione degli Archivi di Stato - Fonti XVI, 1993), 186.
} 
Sicilia invasa il 10 luglio. Entrati a Messina il 16 agosto. Isola occupata in 38 giorni. [...] E' impossibile ora valutare il bottino e il materiale bellico catturato. [...] Nel corso dei combattimenti sono caduti 4.678 italiani, 4.325 tedeschi, 2.376 britannici, 2.811 americani e 490 canadesi $^{16}$.

Il 29 agosto si svolge al Quirinale una riunione per decidere se accogliere, entro la data del 30 fissata dagli alleati, le condizioni stabilite nel testo dell'armistizio cosiddetto "corto", che contiene solamente clausole militari. Il Re afferma che la responsabilità della decisione ricade sul capo del governo Badoglio. Il generale Eisenhower ad Algeri riceve un telegramma del presidente Roosevelt che lo autorizza a procedere con le clausole militari, onde ottenere la firma e poi trasmettere il testo completo ai rappresentanti italiani.

Il 30 agosto il maresciallo Badoglio invia in Sicilia il generale Castellano ${ }^{17}$ per informare gli alleati che l'Italia è disponibile a rompere l'alleanza con la Germania, a prendere tutte le misure contro le forze tedesche per cacciarle al di là delle Alpi e a cessare le ostilità contro le Nazioni unite; nella memoria del ministro degli Esteri Guariglia si afferma, inoltre, che l'Italia avrebbe potuto richiedere l'armistizio solo se "in seguito a sbarchi alleati si cambiassero le attuali condizioni". Il 31 agosto l'aviazione alleata bombarda ancora una volta Pisa, provocando un numero accertato di 1.945 morti. Il 1 settembre è la volta di Napoli che viene bombardata per la centocinquesima volta dall'inizio del conflitto, la città ha subito fino a questo momento 22000 morti e 100000 appartamenti distrutti.

Il giorno 3, mentre gli alleati sbarcano in Calabria, Badoglio riceve in udienza Rudolf Rahn, al quale prima conferma la fedeltà italiana all'alleanza con la Germania e poi gli chiede: "di impedire che le autorità militari tedesche prendano misure inconsulte o addirittura provocatorie [...]. Ho dato la mia parola e la manterrò. Vi prego di avere fiducia" 18 . A distanza di qualche ora, invece, partecipa ad una riunione alla quale prendono parte il ministro della Real Casa Acquarone, della Guerra Sorice, della Marina De Courten, dell'Aeronautica Sandalli ed il generale Ambrosio ai quali, sempre Badoglio, afferma che le trattative per l'armistizio sono in corso (invece si sono di fatto concluse con la firma a Cassibile) e che gli alleati effettueranno ancora piccoli sbarchi in Calabria, successivamente un grosso sbarco vicino a Napoli con 6 divisioni e poi una divisione di paracadutisti giungerà vicino Roma dove nel frattempo saranno concentrate le 6 divisioni. Mentre a Roma si discute ciò, a Cassibile, il generale Castellano, a nome del maresciallo Badoglio, e il generale Bedell Smith, a nome del generale Eisenhower, provvedono a firmare l'armistizio. Secondo quanto dichiarato successivamente dagli alleati, dopo la firma ed il pranzo, il generale Bedell Smith consegna al generale Castellano la cartella contenente le clausole aggiuntive dell'armistizio lungo. MacMillan telegrafa a Churchill: "Le condizioni di armistizio sono state firmate senza emendamenti di nessun genere". Il generale Alexander in un messaggio al Comando supremo britannico annuncia:

Ho passato tutta la scorsa notte in colloqui militari con la parte italiana. Ho messo bene in chiaro con loro che al momento della proclamazione ufficiale dell'armistizio cessiamo di essere nemici ma non diventiamo, ripeto, non diventiamo alleati. Ho dato loro le specifiche indicazioni sulle operazioni da svolgere ${ }^{19}$.

\footnotetext{
${ }^{16}$ Cfr. Mario Avagliano, Il partigiano Montezemolo..., 164.

${ }^{17}$ Giuseppe Castellano, Come firmai l'armistizio di Cassibile (Milano: Mondadori, 1945).

${ }^{18}$ Cfr. Elena Aga Rossi, L'inganno reciproco..., 188.

${ }^{19}$ Cfr. Elena Aga Rossi, L'inganno reciproco..., 190.
} 
Il 6 settembre, a firma avvenuta dell'armistizio, l'aviazione alleata colpisce nuovamente Napoli da dove, il giorno seguente, prendono il largo in missione di guerra, contro le navi alleate, 8 sommergibili, così com'era stato richiesto dagli stessi angloamericani; in effetti i sommergibili italiani non possono sospendere le missioni, dato che ciò rivelerebbe al nemico lo scopo comune, cosicché, alle ore 22.00, nel golfo di Salerno, gli alleati affondano il sommergibile Vella.

A Roma, nella notte, il generale americano Taylor, vice comandante della divisione aerotrasportata, obbliga il maresciallo Badoglio a redigere personalmente un messaggio diretto al generale Eisenhower dove chiede di annullare l'operazione progettata per la conquista di Roma. Badoglio telegrafa:

Dati cambiamenti e precipitare situazione esistenza forze tedesche nella zona di Roma non è più possibile accettare l'armistizio immediato dato che ciò porterà la capitale ad essere occupata ed il governo ad essere sopraffatto dai tedeschi. Operazione Giant 2 non è più possibile dato che io non ho forze sufficienti per garantire gli aeroporti ${ }^{20}$.

L'8 settembre ${ }^{21}$, alle 12, il re riceve la visita dell'ambasciatore germanico Rudolf Rahn al quale comunica, in un momento di totale caos istituzionale, di avere fiducia nel maresciallo Badoglio "Dica al Fuhrer che 1'Italia non capitolerà mai. E' legata alla Germania per la vita e per la morte".

Nello stesso tempo Badoglio affida al generale Rossi un secondo messaggio per il generale Eisenhower per spiegare le ragioni per le quali bisogna annullare l'operazione Giant 2; questa mossa risulterà del tutto inutile perché giungerà ad Algeri quando l'armistizio è già stato annunciato. Eisenhower in risposta alla richiesta di Badoglio di procrastinare l'annuncio ufficiale dell'armistizio comunica:

Ho intenzione di diffondere l'esistenza dell'armistizio all'ora programmata originariamente. $\mathrm{Se}$ voi o qualunque parte delle vostre forze armate mancherete di cooperare come precedentemente concordato, renderò di pubblico dominio in tutto il mondo una documentazione completa relativa a questo affare. Oggi è il giorno $\mathrm{X}$, e mi aspetto che facciate la vostra parte. Non accetto il messaggio di questa mattina che differiva l'armistizio. I vostri rappresentanti accreditati hanno firmato questo accordo con me e la sola speranza dell'Italia dipende dalla vostra adesione a quell'accordo. In base alla vostra pressante rimostranza [...]. Voi avete vicino a Roma truppe sufficienti a garantire la temporanea sicurezza della città [...]. La mancanza da parte vostra nell'adempiere pienamente agli obblighi verso l'accordo firmato avrà le più serie conseguenze per il vostro paese.

\section{Il giorno seguente Churchill comunica a Roosevelt:}

La gente deve essere gradualmente portata a rendersi conto di ciò che noi e il nostro Stato maggiore abbiamo così chiaramente in testa, e cioè la conversione dell'Italia in un partner attivo nei confronti della Germania. Benché non possiamo riconoscere l'Italia come alleato nel pieno senso della parola, ci siamo trovati d'accordo nel fatto che le si debba permettere

\footnotetext{
${ }^{20}$ La lettera è apparsa su Il Messaggero del 7 settembre 2017.

${ }^{21}$ L'8 settembre è da sempre una data molto discussa per le sorti del nostro Paese. in effetti, c'è chi vede in questo giorno la morte della Nazione e chi, come Ernesto Galli della Loggia, il punto di partenza della nuova Nazione.
} 
di guadagnarsi il passaggio e che un appoggio utile contro il nemico non solo sarà aiutato, ma anche ricompensato ${ }^{22}$.

Un dispaccio radio, trasmesso alle ore 1,35 dallo Stato Maggiore Generale, alle unità dipendenti prescrive: "di reagire immediatamente ed energicamente et senza speciali ordini at violenza armata germanica", imponendo, contemporaneamente, che "non deve essere presa iniziativa atti ostili contro germanici".

Il Comando supremo germanico con un comunicato dichiara che le forze armate italiane non esistono più. Eisenhower commenta in merito:

Internamente gli italiani sono stati così deboli e supini che abbiamo avuto poco o nessun aiuto pratico da loro. Comunque, quasi sulla base di un puro bluff abbiamo ottenuto la flotta italiana a Malta e grazie alla resa italiana siamo stati in grado di correre a Taranto e Brindisi dove nessun tedesco era presente. [...] Badoglio vuole incontrarmi ed ha suggerito la Sicilia come luogo dell'incontro. Sto per dirgli che deve venire qui. Egli vuole portare anche qualcuno del suo Stato maggiore, ma non so immaginare che cosa il suo Stato maggiore può comandare in questo momento ${ }^{23}$.

\section{L'inizio di una nuova guerra}

Vittorio Emanuele, stanziatosi a Brindisi, invia un messaggio personale al re d'Inghilterra e al presidente americano per chiedere che al suo governo sia concesso di esercitare i poteri civili oltre che nelle quattro province della Puglia e in Sardegna, anche in Sicilia.

In questo clima sul litorale salernitano aveva inizio lo sbarco alleato, denominato in codice, Operazione Avalanche ${ }^{24}$, affidato al VI corpo d'armata americano ed al X inglese, guidati dal generale Clark. Questa operazione era nata sulla falsa idea di una rapidissima risalita verso Napoli per la conquista della città e soprattutto del porto, ritenuto essenziale ai fini dell'approvvigionamento di un grosso contingente alleato operante nel mediterraneo. Fu scelta la piana di Paestum da dove si godeva una perfetta visuale su tutta l'estensione dell'operazione in modo che poche postazioni di cannoni potevano tenere sotto controllo dalle alture periferiche l'intero arco costiero, non solo le navi in rada ma anche e soprattutto i mezzi da sbarco e da dove, inoltre, per la testa di ponte era facile uscire, in direzione di Napoli, direttamente da Salerno o bypassando da Avellino. I preparativi si avviarono a partire dal 17 agosto in modo molto segreto e solo in un secondo tempo fu comunicato ai comandanti che l'obiettivo era proprio Salerno; la maggioranza dei reparti, gli ufficiali e le truppe furono informati della destinazione quando già erano in mare a poche miglia dalla costa.

Così come dai piani prestabiliti allo scadere del primo minuto del giorno 9 settembre 1943 iniziò il trasbordo delle truppe sui mezzi da sbarco che, appena carichi, manovrarono verso la linea di partenza ad una distanza di 6 miglia dalla costa, seguiti da imbarcazioni cariche di artiglieria antiaerea, mezzi corazzati, munizioni ed armi pesanti.

Tra le 3.35 e le 3.42 la spiaggia a sud della foce del Sele, a ridosso dei templi di Paestum, fu invasa dalle chiglie dei mezzi da sbarco che rasparono l'arenile dove batteva un mare calmo e senza risacca. Rapidamente i portelloni di prua furono abbassati, e le prime ondate di fanti si riversarono in fretta sulla spiaggia cercando di attraversarla fino alla linea

\footnotetext{
${ }^{22}$ Cfr. Cfr. Roosevelt Churchill, Carteggio segreto di guerra... 65.

${ }^{23}$ Cfr. Cfr. Roosevelt Churchill, Carteggio segreto di guerra... 68.

${ }^{24}$ Cfr. Angelo Pesce, Salerno 1943. Operazione Avalanche (Salerno: Cassa di Risparmio Salernitana, 1993).
} 
delle dune costiere; a quel punto la riva si illuminò dalla luce delle fiaccole sparate in aria dalle postazioni litoranee e le mitragliatrici cominciarono a mietere le prime vittime. Il primo vero errore tattico degli alleati fu quello di non effettuare, nelle ore antecedenti lo sbarco, un bombardamento con lo scopo di ottenere, così, una sorpresa tattica. La Luftwaffe, in effetti, allertata da giorni aveva inviato la sedicesima divisione corazzata nella pianura salernitana ed era anche in allerta nell'altro golfo quello di Gaeta; in effetti, si aveva la certezza che ci sarebbe stato ben presto uno sbarco degli angloamericani. Nei momenti dello sbarco al primo avvistamento di soldati nemici si diede inizio ad una serie di mitragliamenti a bassa quota: tra un combattimento di aria ed uno di terra, Capaccio cadde senza opposizione alle 18,15. Contemporaneamente anche a Salerno e Vietri, grazie alla divisione dei Commandos, ed a Maiori a quella dei Rangers era iniziato lo sbarco.

Il 10 e l'11 settembre furono giorni di duri combattimenti; in effetti le quattro divisioni non erano riuscite a penetrare fino in fondo perché bloccate tra Eboli e Battipaglia una, a Molina di Vietri una seconda e sul valico di Chiunzi la terza. La quarta di stanza in una Salerno quasi deserta aveva avuto cammino facile fino al giorno 12 quando venne bloccata sia nei pressi di Fratte che sulla collina del sanatorio, dove i tedeschi continuavano a mantenere ben salda la difesa.

In effetti, il 12 settembre fu un giorno di fondamentali decisioni da prendere: sul fronte tedesco si doveva decidere se sferrare un imponente contrattacco su tutti i fronti e rimandare in mare gli alleati o ritirare le forze e attendere i rinforzi che arrivavano dalla Calabria preparandosi a mantenere l'interno della Piana ed il corridoio interno verso Napoli sulle assi Nocera/Pagani/Chiunzi e Baronissi/Sanseverino; le forze alleate, invece, dal canto loro, si trovavano in una posizione difensiva che aveva creato un blocco per l'operazione Avalanche, che aveva registrato differenti sconfitte, mettendo in seria crisi la testa di ponte fino al giorno 15, facendo balenare in Clark la concreta possibilità di una sconfitta. Lo sganciamento di 1900 paracadutisti, però, dette un nuovo segnale di positività alla testa di ponte tra Agropoli ed Albanella e l'arrivo da Malta delle navi Werspite e Valiant, con sei cacciatorpediniere in rada, portarono un nuovo slancio agli alleati che sferrarono un duro attacco. I tedeschi, di fronte allo strapotere dell'aviazione e dei cannoni navali nemici cominciavano a non poterne più, in quanto la loro contraerea era inadeguata, soprattutto non volevano che le truppe germaniche rimanessero intrappolate nel sud Italia. Iniziarono, così, una immediata ritirata che avrebbe continuato, al tempo stesso ad impedire agli alleati con tutte le forze l'accesso alle vie di comunicazione dirette per Napoli. Infatti, il pomeriggio del 18 settembre la testa di ponte poteva tranquillamente avanzare da Contursi per la valle del Sele, Battipaglia, Olevano, Acerno, Avellino e proseguire per il vero obiettivo che era Napoli, dove, però, le forze una volta oltrepassati gli ostacoli tra Molina e Cava e tra Nocera/Pagani/Sarno riuscirono ad entrare a Napoli solamente il 1 ottobre. L'operazione Valanga, da molti definita una valanga di errori, ebbe la sua conclusione nella giornata del 18 settembre dopo 9 giorni di combattimenti. La quinta sinfonia di Beethoven, scelta dalla BBC come simbolo di vittoria, precedette il giornale radio da Londra che annunciava che anche Salerno era libera e posta sotto il controllo degli alleati.

Dall'altra parte Kesserling, rientrato nelle grazie di Hitler, compiaciuto dalla sua strategia tanto di difesa quanto di attacco, ne aveva approvato il piano di difesa dell'Italia palmo a palmo, metro dopo metro,tanto che lo nominò Comandante di tutte le truppe tedesche a sud del Brennero. Nomina con la quale Kesserling mise in opera il suo personale e diabolico piano di adoperare l'Italia come vero e proprio scannatoio. 
Un Mussolini diverso, tanto nel fisico che nella mente, riottenuta la libertà grazie ad Hitler, proclamava, sotto la pressione dell' antico alleato, a cui nuovamente ha stretto la mano:

Da oggi 15 settembre 1943, assumo nuovamente la suprema direzione del fascismo in Italia. Nomino Alessandro Pavolini alla carica provvisoria di segretario del Pnf che da oggi si chiamerà Partito repubblicano fascista.

Ordino che tutte le autorità militari, politiche, amministrative e scolastiche, nonché tutte quelle che vennero esonerate dalle loro funzioni da parte del governo della capitolazione, riprendano immediatamente i loro posti e i loro uffici.

Ordino l'immediato ripristino di tutte le istituzioni del partito con i seguenti compiti: a) di appoggiate efficacemente e cameratescamente l'esercito germanico che si batte sul territorio italiano contro il comune nemico; b) di dare al popolo immediata, effettiva assistenza morale e materiale; c) di riesaminare la posizione dei membri del Partito in rapporto al loro contegno di fronte al colpo di stato della capitolazione e del disonore, punendo esemplarmente i vili e i traditori;

Ordino la ricostituzione di tutti i reparti e le formazioni speciali della Milizia volontaria per la sicurezza dello Stato.

Da questo momento per l'Italia comincia la terza ed ultima fase della guerra, quella più atroce e controversa in quanto combattuta tra quei fratelli partigiani e repubblichini. Sono solo pochi anni che la storiografia assevera che in Italia, all'indomani dell'armistizio si è combattuta una vera e propria guerra civile. È questo il capitolo finale della lunga storia della Seconda Guerra Mondiale che terminerà con la fine del mese di aprile del 1945. 


\section{Bibliografia}

Aga Rossi Elena. L'inganno reciproco l'armistizio tra l'Italia e gli angloamericani nel settembre 1943. Roma: Pubblicazione degli Archivi di Stato - Fonti XVI, 1993.

Alexander Harold. "The allied Armies in Italy from 3rd september 1943 tho 12th december 1944”, supplemento a The London Gazete.

Avagliano Mario. Il partigiano Montezemolo: storia del capo della resistenza militare nell'Italia occupata. Milano: Dalai editore, 2012.

Castellano Giuseppe. Come firmai l'armistizio di Cassibile. Milano: Mondadori, 1945.

Mac Millan Harold. Diari di guerra. Il Mediterraneo dal 1943 al 1945. Bologna: Il Mulino, 1987.

Mazzetti Massimo. "Gli avvenimenti dell'8 settembre nel quadro della strategia della II Guerra Mondiale". In Atti del Convegno internazionale otto settembre 1943 Ministero della Difesa, Roma, 1983.

Mussolini Benito. Scritti e discorsi di Benito Mussolini. Milano: Hoepli, 1934.

Mussolini, Benito."Discorso di Monaco del 18 settembre 1943”. Meridiano d'Italia illustrato, 12 marzo 1952.

Pavolini Paolo. 1943. La caduta del Fascismo vol 2. Milano: Fratelli Fabbri Editori, 1973.

Pesce Angelo. Salerno 1943. Operazione Avalanche. Salerno: Cassa di Risparmio Salernitana, 1993.

Puntoni, Paolo. Parla Vittorio Emanuele III. Bologna: II Mulino, 1993.

Rainero Romain H. "Gli armistizi di settembre”. In Atti del Convegno internazionale otto settembre 1943 - Ministero della Difesa, Roma, 1983.

Roosevelt Churchill. Carteggio segreto di guerra, Francis Loevenheim, Harold D. Langley e Manfred Jones (a cura di). Milano: Mondadori, 1977.

Rossotto Riccardo. "Verso Cassibile”, Storia Militare n. 108 (2002).

Schroeder Jhon. La caduta di Mussolini e le contromisure tedesche nell'Italia centrale. De Felice Renzo (a cura di). Bologna, 1977.

Tamaro Attilio. Due anni di Storia 1943-1945. Roma: Tosi Editore, 1948.

Zangradi Ruggiero. L'Italia tradita - 8 settembre 1943. Milano: Mursia, 1971. 
Comitato Scientifico per la pubblicazione dei documenti diplomatici italiani $9^{\text {a }}$ Serie: 4 settembre 1939 - 8 settembre 1943, completa in dieci volumi, curati da Mario Toscano (I, II, III, IV, V), Pietro Pastorelli (VI, VII, IX, X) e Giuseppe Vedovato (VIII). 10 Serie: 9 settembre 1943-7 maggio 1948, completa in sette volumi, curati da Pietro Pastorelli (I, III, IV, VI, VII) e Ennio Di Nolfo (II, V). 